\title{
The Efficacy and Safety of Succinylated Atelocollagen and Adenosine for the Treatment of Periorbital Wrinkles*
}

\author{
Dong Jin Ryu ${ }^{1}$, Jin Young Jung ${ }^{1}$, Kee Yang Chung ${ }^{1}$, Hwal Suh ${ }^{2}$, Sang Ho Oh ${ }^{1}$, Ju Hee Lee ${ }^{1 \#}$ \\ ${ }^{1}$ Department of Dermatology and Cutaneous Biology Research Institute, Yonsei University College of Medicine, Seoul, Korea; ${ }^{2}$ De- \\ partment of Medical Engineering, Yonsei University College of Medicine, Seoul, Korea. \\ Email: ${ }^{\#}$ juhee@yuhs.ac
}

Received July $24^{\text {th }}, 2013$; revised August $22^{\text {nd }}, 2013$; accepted August $30^{\text {th }}, 2013$

Copyright (C) 2013 Dong Jin Ryu et al. This is an open access article distributed under the Creative Commons Attribution License, which permits unrestricted use, distribution, and reproduction in any medium, provided the original work is properly cited.

\begin{abstract}
The degradation of structural collagen contributes to the characteristic appearance of wrinkles. The anti-wrinkle effects of a variety of substances have been studied, but the potential anti-wrinkle effects of topical applications of collagen for periorbital wrinkles have not been investigated. To evaluate the effects of topical application of succinylated atelocollagen on periorbital wrinkles and to compare the results of treatment with adenosine, a clinical study on Korean participants was carried out. Each participant's right or left periorbital area was treated with either a solution containing succinylated atelocollagen and adenosine or a solution containing only succinylated atelocollagen for two months. A placebo solution was applied to the opposite periorbital area of each patient's face for two months. Based on objective and subjective measurements of clinical improvement, the assessment scores for treated sites were statistically significantly higher than scores for placebo sites after two months of treatment. Analysis of silicone casts of periorbital wrinkles demonstrated partial effects of succinylated atelocollagen on periorbital wrinkles. However, we did not observe any effects of adenosine on periorbital wrinkles. Succinylated atelocollagen may be an effective treatment option for periorbital wrinkles, but further studies including a longer treatment period and larger subject group are needed to verify these results.
\end{abstract}

Keywords: Adenosine; Succinylated Atelocollagen; Periorbital Wrinkles

\section{Introduction}

Skin aging manifests as changes in skin thickness, color, elasticity, vascular dilatation and wrinkles. Epidermal atrophy and the degeneration of dermal collagen and elastic fibers lead to the formation of wrinkles and fine lines. Due to mimetic muscle movement and the remodeling of bony tissues, deeper and more prominent wrinkles occur with aging [1]. Sunlight, smoking and pollutants increase the production of collagenase, and an enzyme breaks down collagen through a signal transduction cascade, resulting in the acceleration of wrinkle formation [2]. In the field of dermatology, there is increasing demand for effective, convenient medications and cosmetics that prevent or treat wrinkles and that have few side effects.

Studies on a variety of agents that may be effective for

\footnotetext{
*This research was supported by the grants from Dalim Corporation (Seoul, Korea) 4-2008-0333 and 1-2011-0006. The authors have no conflicts of interest to report.

${ }^{\#}$ Corresponding author.
}

treating and preventing wrinkles are ongoing, with retinoids, vitamin $\mathrm{C}$ and adenosine considered especially effective [1]. Several anti-oxidants, including vitamin E, ferulic acid, co-enzyme Q10, idebenone, green tea, silymarin and pycnogenol, are incorporated into skin care products [1]. Asiaticoside, phosphatidylserine, ginseng saponins isolated from red ginseng, and K6PC-5, a novel sphingosine kinase activator, are under continued study, having demonstrated antioxidative effects and wrinkle improvement [3-5]. However, the potential anti-wrinkle effects of topical applications of collagen, a component of the dermis, have not been investigated.

Collagen is a fundamental component of the dermis, and type I collagen makes up $80 \%$ of the collagen in the skin [6]. As aging progresses, fragmented type I collagen fibrils become prominent and disrupt the mechanical properties of the skin, leading to wrinkle formation [7]. Therefore, supplying collagen, the major component of the skin's extracellular substrate, may offer an effective fundamental treatment for wrinkles. However, collagen 
is insoluble in neutral solution and has a large molecular weight $(300 \mathrm{kDa})$, so it is not sure that collagen penetrates into the skin. In addition, when collagen is kept at room temperature, it becomes denatured and loses its triple helix structure, rendering it ineffective. Due to these limitations, direct injections of small collagen particles dispersed in distilled water are often used by dermatolo- gists rather than topical applications.

Among the succinylated atelocollagen used in this research, the telopeptide on both ends, which causes immune reactions, was eliminated to minimize the immune reactions in human body. Furthermore, the atelocollagen was treated with succinic anhydride to electrically induce an anionic status that improves solubility in water or neutral solution. We performed a double blind, randomized, prospective, split-face clinical study on Korean participants with periorbital wrinkles to evaluate the effects of topical application of succinylated atelocollagen on periorbital wrinkles. We compared the results of topical atelocollagen treatment to the results of treatment with adenosine, which is an approved anti-wrinkle treatment ingredient.

\section{Methods}

\subsection{Patients}

The study protocol was approved by the Institutional Review Board of Severance Hospital, Yonsei University College of Medicine, Seoul, Korea and informed consent was obtained from each participant. Thirty-two female participants (mean age 49.1; age range 32 - 59 years) with periorbital wrinkles were enrolled in a double-blind, randomized, prospective, split-face clinical study. The participants were stratified based on a baseline facial grading scale, ranging from 0 to $5(0$, no evidence of lines or wrinkles to 5 , many deep lines below the eye with several coarse wrinkles that extend into the cheek area) [8]. Exclusion criteria included a history of keloid scarring or other skin diseases, any laser procedures or isotretinoin use within six months of study initiation, pregnancy, or systemic diseases that are known to affect metabolism. Patients with facial scale grades of 0 or 1 were also excluded.

\subsection{Treatments}

One side of each participant's face (periorbital area) was randomly assigned to be treated with either a solution containing succinylated atelocollagen and adenosine (sodium hyaluronate, L-lysine, succinylated atelocollagen, adenosine) (Group A), or a solution containing only succinylated atelocollagen (sodium hyaluronate, L-lysine, succinylated atelocollagen) (Group B). A placebo (sodium hyaluronate, L-lysine) solution (Group P) was applied to the other side of each patient's face. Compositions of the test and placebo solutions are summarized in Table 1. Subjects were instructed to apply the treatment and placebo solutions twice a day for two months on each side of the face in the periorbital area. Patients were instructed to avoid the use of any bleaching or antiwrinkle agents during the course of treatment. Skin biopsies were obtained from eight subjects, four from each group, for histological analyses of both periorbital areas before and after the two-month-treatment period.

\subsection{Assessment of Clinical Effects}

Before treatment and after one and two months of treatment, digital photographs were taken of each patient using identical camera settings and lighting conditions. Two dermatologists, blinded to the patient's treatment, evaluated clinical improvements by studying these photographs, and the participants themselves subjectively evaluated improvements at the same time points. Both patients and clinicians followed a five-point grading scale: $0=$ no improvement, $1=1 \%-25 \%, 2=26 \%-$ $50 \%, 3=51 \%-75 \%, 4=76 \%-100 \%$ improvement.

We used silicone casts (Replica ${ }^{\mathrm{TM}}$, Cuderm Inc., Dallas, TX, USA) of the participants' periorbital wrinkles to assess skin texture before and after the two-month treatment period. An adhesive ring locator was placed against the periorbital area and filled with pre-mixed resin so that the resin overflowed $2-3 \mathrm{~mm}$ onto the cardboard surface. After the resin set and the cast was peeled from the skin surface, the foam adhesive spacer layer was separated from the cardboard frame and discarded. The casts were labeled with the date, initials and group and stored in a paper envelope until analysis. For analysis, a collimated light source was directed at a $25^{\circ}$ angle from the plane of

Table 1. Compositions of the test and placebo solutions.

\begin{tabular}{cccc}
\hline Ingredients $(\mathrm{gm} / 30 \mathrm{ml})$ & Group A (collagen + adenosine) & Group B (collagen) & Group P (placebo) \\
\hline Sodium hyaluronate & 0.12 & 0.12 & 0.12 \\
L-lysine & 0.00006 & 0.00006 & 0.00006 \\
Succinylated atelocollagen & 0.12 & 0.12 & - \\
Adenosine & 0.012 & - & - \\
\hline
\end{tabular}


the cast surface. Each cast was placed in a holder that fixed the direction of the tab position of the cast.

The textures of shadows produced by oblique lighting of the negative casts were analyzed via two assay methods. The first method involved measuring the luminance along a set of 10 equal-length parallel lines (passes) running across the cast parallel to the lighting direction. Variations in luminance were treated as indicators of surface roughness $\mathrm{Rz}$, the average maximum difference in the luminance value for five equal-length segments in each of the ten lines traversing the sample; and Ra, the average deviation of the luminance curve about the mean luminance for the same ten lines, with "R" parameters reported in units of brightness (gray levels) ranging from 0 to 255 and increase with increasing roughness; FNum, number markers indicative of fine and coarse lines per $\mathrm{mm}$; and IDL, the integrated developed length of the luminance traces of the ten scan lines. The IDL is the total length of the luminance lines as a proportion of the straight-line distance and it increases with the roughness of the surface. For the second assay method, the cast image area was divided into ten equal width bands or subareas. Shadow-like features were distinguished in each of these bands when their luminance values were less than the detection threshold. Four parameters were determined from the detected features: spacing, or the mean distance in millimeters between adjacent detected features, which increases with disappearance of wrinkles; breadth, or the average breadth of the detected features in millimeters, which is proportional to the depth of the wrinkles producing shadows and decreases as wrinkles become shallower; shadows, or the percent of the sampled replica area with luminance values less than the detection threshold, which the relative area of shadow cast by the wrinkles in the cast (shadows decrease with smoothing of the skin); and NumWr, or the total number of shadowy features detected in the ten bands or sub-areas, which decreases with smoothing of the skin.

\subsection{Assessment of Histological Changes}

Skin biopsies were taken from eight volunteers before treatment and after the two-month-treatment period using a $3 \mathrm{~mm}$ biopsy punch. At the first visit, specimens were obtained from crow's feet at a $1.5 \mathrm{~cm}$ distance from the lateral margin of both eyes. The biopsies performed after the two-month-treatment period were taken directly next to the previous biopsy sites. The specimens were fixed in formalin and embedded in paraffin. Skin samples were stained with hematoxylin and eosin to measure the epidermal thickness, Masson trichrome to evaluate changes in dermal collagen, monoclonal anti-human procollagen type I antibody (Abcam Inc., Cambridge, MA, USA) to demonstrate changes in collagen formation and monoclonal anti-human Ki-67 antibody (Abcam Inc.) to eva- luate changes in cellular proliferation in the skin. Images of each section were taken at a magnification of X200 with a 12.5 megapixel digital camera (DP70, Olympus Optical Co., Tokyo, Japan) connected to a light microscope (BX40, Olympus Optical Co.). The images were analyzed by MetaMorph (Molecular Devices, Sunnyvale, CA, USA).

\subsection{Statistical Analysis}

Statistical analysis was performed using the Wilcoxon signed-rank and rank-sum tests for evaluation of objective and subjective improvement scores and histological changes. One sample t-test and an independent group $\mathrm{t}$-test were used to evaluate changes in the casts. $P$-values less than 0.05 were considered statistically significant and $P$-values less than 0.10 were considered directionally significant.

\section{Results}

\subsection{Degree of Clinical Improvement}

Twenty-eight of 32 participants completed the twomonth study. Four patients dropped out for personal reasons without experiencing any side effects. The numbers of patients in each group were 15, 13 and 28 in groups A, $\mathrm{B}$ and $\mathrm{P}$ respectively. The mean clinical improvement scores, evaluated by two blinded dermatologists, at the one-month treatment assessment were $1.53 \pm 0.64,1.08 \pm$ 0.86 and $0.57 \pm 0.50$ in groups $\mathrm{A}, \mathrm{B}$ and $\mathrm{P}$ respectively. The mean clinical improvement scores at the two-month treatment assessment were $2.27 \pm 0.96,2.31 \pm 1.25$ and $0.96 \pm 0.74$ in groups A, B and P respectively (Figure 1). A significant difference in clinical improvement was observed between groups A and P $(P=0.0005)$, but we did not observe a significant difference between groups $\mathrm{B}$ and $\mathrm{P}$ at the one-month treatment assessment $(P>$

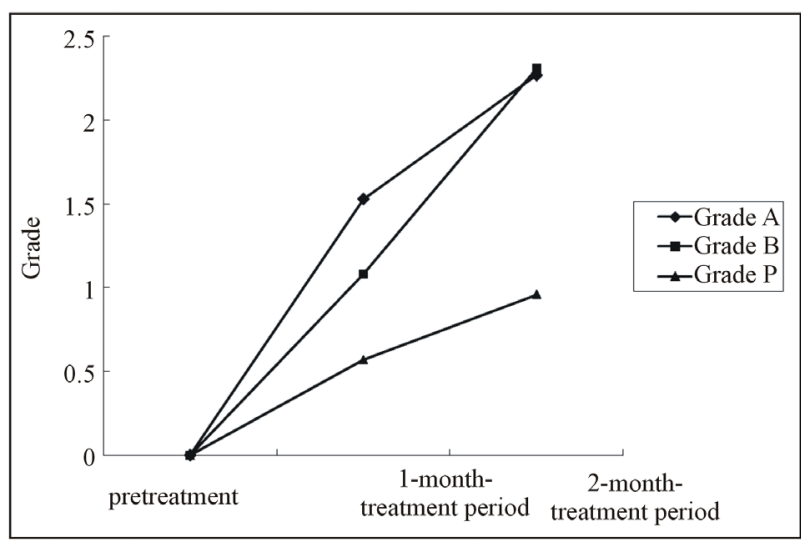

Figure 1. Mean clinical improvement scores assessed by physicians (grading scale: $0=$ no improvement, $1=1 \%$ $25 \%, 2=26 \%-50 \%, 3=51 \%-75 \%, 4=76 \%-100 \%$ improvement). 
0.05). Significant differences were observed between groups $\mathrm{A}$ and $\mathrm{P}$, and between groups $\mathrm{B}$ and $\mathrm{P}$ at the twomonth treatment assessment $(P=0.0005, P=0.0039)$. There were no significant differences between groups $\mathrm{A}$ and $\mathrm{B}$ at the one-month or two-month treatment assessments $(P>0.05)$.

The improvement scores assessed by the physicians and by participants were similar (Figure 2). However, at the one-month treatment assessment, no significant differences were observed among the three groups $(P>0.05)$ by patients. At the two-month treatment assessment, participant evaluations were significantly different between groups A (Figure 3) and $\mathrm{P}$ (Figure 4) $(P=0.01)$, and between groups $\mathrm{B}$ (Figure 5) and $\mathrm{P}(P=0.001)$. There were no significant differences between groups A and $\mathrm{B}$ at the two-month treatment assessment $(P>0.05)$. The

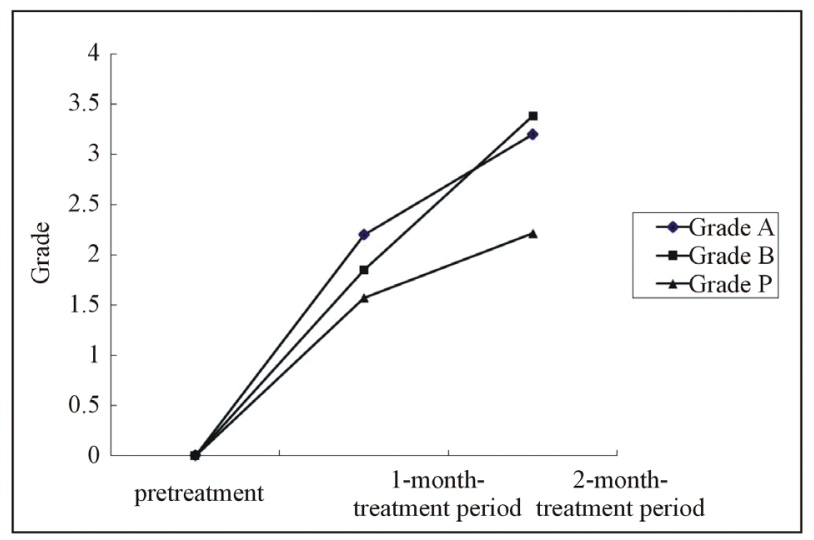

Figure 2. Mean clinical improvement scores assessed by participants (grading scale: $\mathbf{0}=$ no improvement, $1=1 \%$ $25 \%$, $2=26 \%-50 \%, 3=51 \%-75 \%, 4=76 \%-100 \%$ improvement).
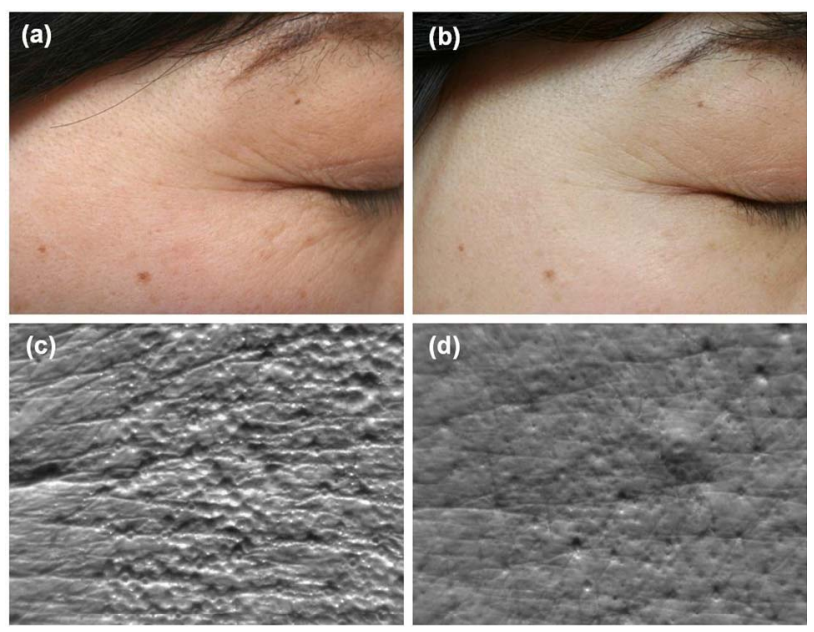

Figure 3. Comparison of clinical improvement of periorbital wrinkles and cast images of representative subject in group A before treatment (a) and two months after the treatment (b). Cast images before treatment (c) and two months after the treatment (d).
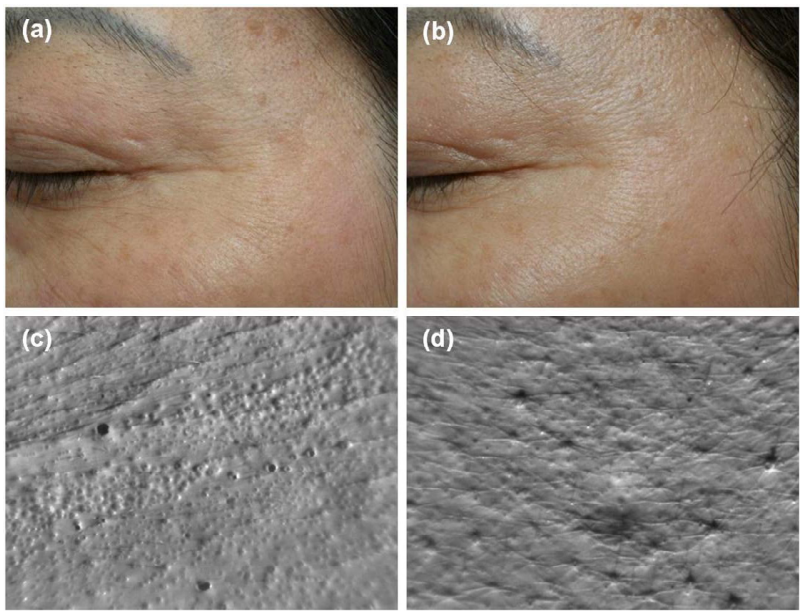

Figure 4. Comparison of clinical improvement of periorbital wrinkles and cast images of representative subject in group $P$ before treatment (a) and two months after the treatment (b). Cast images before treatment (c) and two months after the treatment (d).
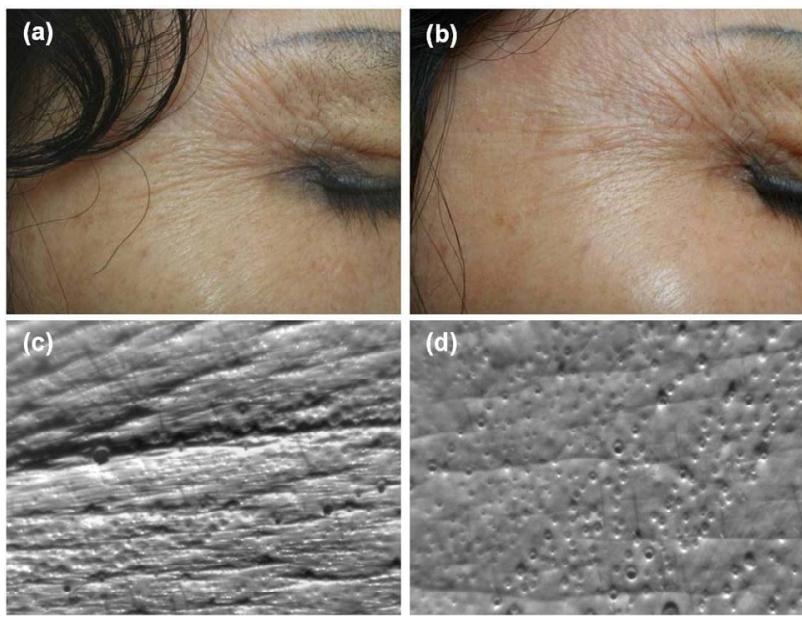

Figure 5. Comparison of clinical improvement of periorbital wrinkles and cast images of representative subject in group B before treatment (a) and two months after the treatment (b). Cast images before treatment (c) and two months after the treatment (d).

treatments were well tolerated and there were no remarkable complications for any of the participants.

\subsection{Cast Image Analysis}

A total of 112 casts were evaluated. The casts represented treated sites for groups A and B, and placebo sites for group $\mathrm{P}$ at two visit times: baseline (BL) and after the two-month treatment period (V2). The results are summarized in Table 2. Changes from baseline were calculated by subtracting each subject's BL values from the appropriate V2 values. Table 3 summarizes the mean changes from baseline for groups A, B and P. Changes from baseline were significant for three measured pa- 
Table 2. The results of cast image analysis at baseline (BL) and after two months of treatment (V2) (Group A, succinylated atelocollagen + adenosine; group $\mathbf{B}$, succinylated atelocollagen; group $\mathbf{P}$, placebo).

\begin{tabular}{|c|c|c|c|c|c|c|}
\hline & \multicolumn{2}{|c|}{ Group A } & \multicolumn{2}{|c|}{ Group B } & \multicolumn{2}{|c|}{ Group P } \\
\hline & BL & $\mathrm{V} 2$ & BL & $\mathrm{V} 2$ & $\mathrm{BL}$ & $\mathrm{V} 2$ \\
\hline $\mathrm{Rz}$ & $128.7 \pm 38.0$ & $109.6 \pm 23.9$ & $139.5 \pm 32.6$ & $113.0 \pm 28.5$ & $144.7 \pm 24.2$ & $126.8 \pm 32.7$ \\
\hline $\mathrm{Ra}$ & $24.5 \pm 8.2$ & $19.7 \pm 4.9$ & $26.9 \pm 8.3$ & $20.5 \pm 6.5$ & $27.9 \pm 6.1$ & $24.3 \pm 7.1$ \\
\hline FNum & $0.476 \pm 0.093$ & $0.439 \pm 0.113$ & $0.399 \pm 0.098$ & $0.386 \pm 0.109$ & $0.469 \pm 0.112$ & $0.469 \pm 0.111$ \\
\hline IDL & $6.95 \pm 2.64$ & $5.48 \pm 1.55$ & $6.84 \pm 2.00$ & $5.28 \pm 1.67$ & $6.93 \pm 1.49$ & $5.88 \pm 1.66$ \\
\hline Spacing & $1.948 \pm 1.381$ & $1.946 \pm 1.053$ & $1.840 \pm 1.259$ & $2.239 \pm 1.177$ & $1.389 \pm 0.325$ & $2.005 \pm 1.288$ \\
\hline Breadth & $0.188 \pm 0.030$ & $0.198 \pm 0.042$ & $0.247 \pm 0.058$ & $0.206 \pm 0.042$ & $0.222 \pm 0.043$ & $0.216 \pm 0.039$ \\
\hline Shadows & $4.2 \pm 3.6$ & $3.1 \pm 2.0$ & $5.4 \pm 3.7$ & $2.7 \pm 2.7$ & $5.8 \pm 3.8$ & $4.4 \pm 3.2$ \\
\hline
\end{tabular}

Table 3. Mean changes from baseline in groups $A, B$ and $P$, and treatment effects calculated by subtracting value of group $P$ from value of group A or B. (Group A; succinylated atelocollagen + adenosine, group B; succinylated atelocollagen, group P; placebo.).

\begin{tabular}{|c|c|c|c|c|c|}
\hline & \multicolumn{3}{|c|}{ Mean changes from baseline } & \multicolumn{2}{|c|}{ Treatment effects } \\
\hline & Group A & Group B & Group P & Group A & Group B \\
\hline $\mathrm{Rz}$ & $-19.1 \pm 40.5^{* *}$ & $-26.5 \pm 37.1^{*}$ & $-17.8 \pm 27.7^{*}$ & $9.2 \pm 51.4$ & $-20.8 \pm 41.3^{* *}$ \\
\hline $\mathrm{Ra}$ & $-4.8 \pm 8.4^{*}$ & $-6.4 \pm 8.2^{*}$ & $-3.6 \pm 6.5^{*}$ & $1.1 \pm 10.5$ & $-5.3 \pm 9.0^{* *}$ \\
\hline FNum & $-0.038 \pm 0.096$ & $-0.014 \pm 0.117$ & $0.001 \pm 0.089$ & $-0.035 \pm 0.129$ & $-0.018 \pm 0.134$ \\
\hline IDL & $-1.47 \pm 2.80^{* *}$ & $-1.56 \pm 2.82^{* *}$ & $-1.04 \pm 1.59^{*}$ & $0.33 \pm 3.11$ & $-1.39 \pm 2.85$ \\
\hline Spacing & $-0.002 \pm 1.665$ & $0.372 \pm 1.768$ & $0.623 \pm 1.193^{*}$ & $-0.615 \pm 2.188$ & $-0.334 \pm 1.978$ \\
\hline Breadth & $0.01 \pm 0.056$ & $-0.041 \pm 0.059^{*}$ & $-0.006 \pm 0.045$ & $0.008 \pm 0.063$ & $-0.026 \pm 0.069$ \\
\hline Shadows & $-1.1 \pm 4.0$ & $-2.7 \pm 3.3^{*}$ & $-1.4 \pm 3.2^{*}$ & $0.3 \pm 4.3$ & $-1.2 \pm 2.8$ \\
\hline NumWr & $-14.1 \pm 57.2$ & $-27.3 \pm 48.2^{* *}$ & $-11.2 \pm 42.0$ & $-4.1 \pm 66.3$ & $-14.8 \pm 49.4$ \\
\hline
\end{tabular}

${ }^{*} P<0.05,{ }^{* *} P<0.10$.

rameters in Group A (Figure 3) and six parameters in Group B (Figure 5). The changes for both groups represented improvements in texture (increased smoothness), with decreases from baseline for Rz, Ra and IDL (both groups A and B), Breadth, Shadows and NumWr (Group $\mathrm{B}$ only). In group $\mathrm{P}$, changes from baseline were significant for five measured parameters (Figure 4). The changes represented improvements in texture: decreases from baseline for Rz, Ra, IDL and Shadows parameters, with an increase in the Spacing parameter.

Similarly, the differences between treated and placebo site pairs (treatment effects) were calculated by subtracting the appropriate baseline corrected values for each subject. These results are shown in Table 3 . Treatment effects were directionally significant for two parameters for group B only. Significant treatment effects for Rz and
Ra were in the direction of active smoother compared to group $\mathrm{P}$.

Groups A and B were also compared. A direct comparison of group A and B treatment effects confirmed that the differences in $\mathrm{Ra}$ parameters between groups were directionally significant, with group B smoother than group A $(P=0.0923)$.

\subsection{Histological Changes}

Changes in histological features between baseline and after two months of treatment are shown in Table 4. Changes from baseline were calculated by subtracting each subject's BL values from the V2 values.

Epidermal thickness increased after two months of treatment compared with values before treatment in all 
Table 4. Quantitative changes in histological features between baseline and after two months of treatment. (Group A; succinylated atelocollagen + adenosine, group B; succinylated atelocollagen, group P; placebo.).

\begin{tabular}{cccc}
\hline Change & Group A & Group B & Group P \\
\hline Epidermal thickness $(\mu \mathrm{m})$ & $10.23 \pm 7.60$ & $13.15 \pm 5.65$ & $6.96 \pm 10.15$ \\
Collagen (OD) & $707.07 \pm 1354.46$ & $873.87 \pm 331.01$ & $756.72 \pm 476.35$ \\
Ki-67 (OD) & $224.28 \pm 102.03^{*}$ & $181.49 \pm 283.44$ & $-102.47 \pm 266.31$ \\
Procollagen type I (OD) & $346.29 \pm 570.80$ & $406.87 \pm 665.45$ & $529.29 \pm 618.73$ \\
\hline
\end{tabular}

OD, optical density. ${ }^{*} P<0.05$.

groups. The periorbital areas treated with succinylated atelocollagen and adenosine containing solution or only succinylated atelocollagen containing solution showed greater increases in epidermal thickness. However, there were no significant differences among the three groups $(P>0.05)$.

Quantitative image analyses of pre- and post-treatment biopsies revealed that after the two-month treatment period, the quantities of collagen fibers of each group had increased compared with baseline (Table 4). However, there were no significant differences among the three groups $(P>0.05)$.

Quantitative image analyses of Ki-67 expression on pre- and post-treatment biopsies showed an increase after the two-month treatment period in groups A and B. However, there was a decrease in the optical density of Ki-67 expression in group P (Table 4). There was a significant difference between groups $\mathrm{A}$ and $\mathrm{P}(\mathrm{P}=0.0455)$, but there were no significant differences between groups $\mathrm{A}$ and $\mathrm{B}$ or between groups $\mathrm{B}$ and $\mathrm{P}(P>0.05)$ (Figure 6).

Immunohistochemical staining of procollagen type I expression revealed that procollagen expression was increased in all groups. Quantitative analyses of procollagen type I expression showed that the changes from baseline were $346.3 \pm 570.8,406.9 \pm 665.5$ and $529.3 \pm$ 618.7 in groups A, B, and $\mathrm{P}$ respectively. However, there were no significant differences among the three groups $(P>0.05)$.

\section{Discussion}

Wrinkle formation represents both photoaging and chronological aging. Periorbital wrinkles are generated early in the formation of wrinkles. At the molecular level, ultraviolet (UV) irradiation activates cytokine receptors and other growth factors on the surface of fibroblasts and keratinocytes. Activated receptors and growth factors stimulate transcription factor AP-1 through a signal transduction cascade, and AP-1 stimulates the transcription of matrix metalloproteinase (MMP) genes. MMPs break down collagen, the main component of the dermal extracellular matrix, and other structural proteins [2]. The
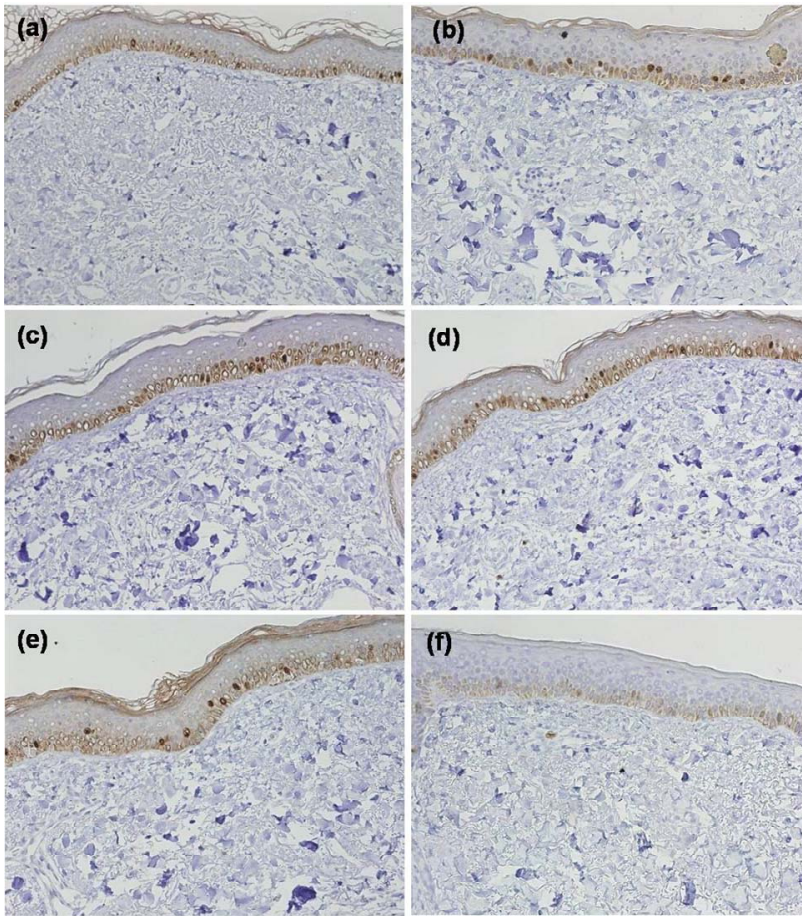

(f)

Figure 6. Immunohistochemical staining of Ki-67 expression (original magnification X200). (a) Before treatment and (b) after two months of treatment using succinylated atelocollagen and adenosine-containing solution; (c) before treatment and (d) after two months of treatment using succinylated atelocollagen-containing solution; (e) before treatment and (f) after two months of treatment using placebo solution.

activation of MMP-1 and MMP-9 leads to the partial degradation of collagen, which regulates procollagen type I synthesis and down-regulates procollagen type I formation [9]. AP-1 stimulated by UV irradiation also interferes with collagen synthesis through down-regulation of procollagen type I gene expression [10]. Collagen provides skin with its tensile strength, so the degradation of structural collagen and reduced collagen synthesis are likely to be major contributors to the characteristic appearance of wrinkles [11]. In chronological aging, molecular features similar to those in photoaging have been identified [2]. 
As mentioned above, some substances have been proven to repair and prevent wrinkles. Many controlled studies have demonstrated that topical applications of retinoids reduce the severity of wrinkles [12]. All-transretinoic acid, a first-generation retinoid, induces production of procollagen types I and III through induction of TGF- $\beta$, a key player in fibroblast collagen synthesis [11]. Increases in levels of collagen types I and III provide resiliency and strength to skin. Another constituent, adenosine, has also been shown to be effective in treating wrinkles [13]. Adenosine acts as a regulator of cellular and organ function, and interacts with a family of $4 \mathrm{G}$ protein-coupled receptors, $\mathrm{A}_{1}, \mathrm{~A}_{2 \mathrm{~A}}, \mathrm{~A}_{2 \mathrm{~B}}$ and $\mathrm{A}_{3}$. After binding to $\mathrm{A}_{2 \mathrm{~A}}$ receptors, collagen production is stimulated by fibroblasts via a MEK-1/MAPK-mediated pathway and collagen degradation is reduced by down-regulation of MMP expression [14].

Increased collagen synthesis and decreased collagen degradation are the keystones of strategies for preventing or improving the appearance of wrinkles. However, there are no reported clinical studies that evaluate the effect of topical collagen on periorbital wrinkles. Collagen enhances the attachment and proliferation of fibroblasts [15]. Fibroblasts bind to intact collagen fibrils via cell surface integrin receptors and this binding creates dynamic mechanical tension within fibroblasts and maintains their shape. Mechanical tension and cell shape are critical for proper cellular function and the synthesis of procollagen fibrils [7]. Recently, there has been an increase in the use of collagen for the treatment of chronic wounds [16].

In this study, we investigated the efficacy of topical application of succinylated atelocollagen-containing solution and compared these effects with the effects of adenosine on periorbital wrinkles. The succinylated atelocollagen-containing solution that we tested was composed of succinylated atelocollagen, L-lysine, sodium hyaluronate and purified water. The size of the succinylated atelocollagen molecule is $300 \mathrm{~nm} \times 2.4 \mathrm{~nm}$. The telopeptide consists of 12 - 27 amino acids in a collagen molecule, and the sequence of the telopeptide is different between individuals. Complete removal of the telopeptide helps prevent immune reactions and reduces the size of the molecule [17]. Succinylated atelocollagen was treated with succinic anhydride to create an electrically anionic surface that improves solubility in water or neutral solution. L-lysine has been widely used as a cell adhesion agent and mediator for drug delivery. Because L-lysine has polycationic sites, it adheres to anionic sites on the cell surface and the succinylated atelocollagen. Therefore, combining the succinylated atelocollagen with L-lysine increases skin penetration. Brown et al. [18] showed that hyaluronic acid, which has hydrophilic properties and a large molecular weight (360 - $400 \mathrm{kDa})$, can be absorbed from the surface of intact skin by passive diffusion and active transport and be observed in the deeper layers of epidermis and dermis. Succinylated atelocollagen has better solubility and similar molecular weight so it may be absorbed through intact human skin. Hyaluronic acid is also a component of the extracellular matrix and has the ability to induce fibroblasts to proliferate and to produce more collagen [19]. In addition, succinylated atelocollagen and hyaluronic acid can hold water within their molecules. Interspersed water has a bulking effect and may improve periorbital wrinkles.

Based on the objective measurements of clinical improvement, groups A and B showed more significant improvement than group $\mathrm{P}$ after a two-month treatment period. However, we did not observe any significant effects after two months of adenosine treatment. The subjective assessments of improvement by the participants were similar to the objective assessments of clinical improvement. In the analysis of the casts, the treated sites for both groups A and B exhibited consistent smoothing of the periorbital wrinkles. In paired comparisons with group $\mathrm{P}$, only group $\mathrm{B}$ showed directionally significant improvement for two parameters of roughness. Although the differences were not statistically significant, other parameters were in the direction of active smoother compared to group P. It was confirmed that only one parameter of roughness $(\mathrm{Ra})$ in group $\mathrm{B}$ was lower than in Group A $(P=0.0923)$. Overall, the results suggest that the treatment effect in group B was somewhat superior to the effects in groups A or P.

Analysis of histological features revealed increased epidermal thickness, collagen and procollagen type I density with treatment in all groups. There were no significant differences among the three groups. The density of Ki-67 in group A was significantly higher than that of group P. Ki-67 protein is a cellular marker for proliferation and can be exclusively detected within the cell nucleus during interphase [20]. It is thought that adenosine, which was included in group A's treatment, may affect the proliferation of keratinocytes. However, we did not observe proliferation of dermal fibroblasts with Ki-67 staining. This may be due to the short treatment time or the low penetration rate of the treatment solution. We were not able to distinguish the effects of succinylated atelocollagen or adenosine on histological features. The lack of significant differences among the three groups may be due to the small number of subjects (each group, $\mathrm{n}=4$ ) and relatively short duration of treatment.

Although degree of clinical improvement showed antiwrinkle efficacy of succinylated atelocollagen, the objective measures such as cast image analysis and histologycal changes did not support it sufficiently. Analysis of cast images showed significant improvement on some parameters between the areas treated with succinylated 
atelocollagen-treated and the areas treated with placebo. However, we did not observe any effects of adenosine on periorbital wrinkles. This may be due to the small number of subjects or to the short treatment period.

\section{Conclusion}

The results of our study show that succinylated atelocollagen may be an effective treatment option for periorbital wrinkles, but further studies with a longer treatment period and larger subject group are needed to verify these results.

\section{REFERENCES}

[1] L. Baumann, "Skin Aging and Its Treatment," The Journal of Pathology, Vol. 211, No. 2, 2007, pp. 241-251. doi:10.1002/path.2098

[2] G. J. Fisher, S. Kang, J. Varani, Z. Bata-Csorgo, Y. Wan, S. Datta and J. J. Voorhees, "Mechanisms of Photoaging and Chronological Skin Aging," Archives of Dermatology, Vol. 138, No. 11, 2002, pp. 1462-1470. doi:10.1001/archderm.138.11.1462

[3] A. Chiu and A. B. Kimball, "Topical Vitamins, Minerals, and Botanical Ingredients as Modulators of Environmental and Chronological Skin Damage," British Journal of Dermatology, Vol. 149, No. 4, 2003, pp. 681-691. doi:10.1046/j.1365-2133.2003.05540.x

[4] H. Y. Park, J. K. Youm, M. J. Kwon, B. D. Park, S. H. Lee and E. H. Choi, "K6PC-5, a Novel Sphingosine Kinase Activator, Improves Long-Term Ultraviolet LightExposed Aged Murine Skin," Experimental Dermatology, Vol. 17, No. 10, 2008, pp. 829-836. doi:10.1111/j.1600-0625.2008.00708.x

[5] Y. G. Kim, M. Sumiyoshi, M. Sakanaka and Y. Kimura, "Effects of Ginseng Saponins Isolated from Red Ginseng on Ultraviolet B-Induced Skin Aging in Hairless Mice," European Journal of Pharmacology, Vol. 602, No. 1, 2009, pp. 148-156. doi:10.1016/j.ejphar.2008.11.021

[6] J. Varani, M. K. Dame, L. Rittie, S. E. G. Fligiel, S. Kang, G. J. Fisher and J. J. Voorhees, "Decreased Collagen Production in Chronologically Aged Skin: Roles of AgeDependent Alteration in Fibroblast Function and Defective Mechanical Stimulation," The American Journal of Pathology, Vol. 168, No. 6, 2006, pp. 1861-1868. doi:10.2353/ajpath.2006.051302

[7] G. J. Fisher, T. Quan, T. Purohit, Y. Shao, M. K. Cho, T. He, J. Varani, S. Kang and J. J. Voorhees, "Collagen Fragmentation Promotes Oxidative Stress and Elevates Matrix Metalloproteinase-1 in Fibroblasts in Aged Human Skin," The American Journal of Pathology, Vol. 174, No. 1, 2009, pp. 101-114. doi:10.2353/ajpath.2009.080599

[8] D. L. Bissett, J. E. Oblong and C. A. Berge, "Niacinamide: A B Vitamin that Improves Aging Facial Skin Appearance," Dermatologic Surgery, Vol. 31, No. s1, 2005, pp. 860-865.

[9] J. Varani, D. Spearman, P. Perone, S. E. Fligiel, S. C. Datta, Z. Q. Wang, Y. Shao, S. Kang, G. J. Fisher and J. J.
Voorhees, "Inhibition of Type I Procollagen Synthesis by Damaged Collagen in Photoaged Skin and by Collagenase-Degraded Collagen in Vitro," The American Journal of Pathology, Vol. 158, No. 3, 2001, pp. 931-942. doi:10.1016/S0002-9440(10)64040-0

[10] G. J. Fisher, S. Datta, Z. Wang, X. Y. Li, T. Quan, J. H. Chung, S. Kang and J. J. Voorhees, "c-Jun-Dependent Inhibition of Cutaneous Procollagen Transcription Following Ultraviolet Irradiation is Reversed by All-Trans Retinoic Acid," The Journal of Clinical Investigation, Vol. 106, No. 5, 2000, pp. 663-670. doi:10.1172/JCI9362

[11] M. Singh and C. E. Griffiths, "The Use of Retinoids in the Treatment of Photoaging," Dermatologic Therapy, Vol. 19, No. 5, 2006, pp. 297-305. doi:10.1111/j.1529-8019.2006.00087.x

[12] M. Samuel, R. C. Brooke, S. Hollis and C. E. M. Griffiths, "Interventions for Photodamaged Skin," Cochrane Database System Review, Vol. 25, No. 1, 2005, CD001782.

[13] M. L. Abella, "Evaluation of Anti-Wrinkle Efficacy of Adenosine-Containing Products Using the FOITS Technique," International Journal of Cosmetic Science, Vol. 28, No. 6, 2006, pp. 447-451. doi:10.1111/j.1467-2494.2006.00349.x

[14] E. S. Chan, P. Fernandez, A. A. Merchant, et al., "Adenosine $A_{2 A}$ Receptors in Diffuse Dermal Fibrosis: Pathogenic Role in Human Dermal Fibroblasts and in a Murine Model of Scleroderma," Arthritis \& Rheumatism, Vol. 54, No. 8, 2006, pp. 2632-2642. doi:10.1002/art.21974

[15] S. Srivastava, S. D. Gorham and J. M. Courtney, "The Attachment and Growth of an Established Cell Line on Collagen, Chemically Modified Collagen, and Collagen Composite Surfaces," Biomaterials, Vol. 11, No. 3, 1990, pp. 162-168. doi:10.1016/0142-9612(90)90149-K

[16] J. U. Shin, Y. J. Choi, M. R. Roh, K. Y. Chung and H. Suh, "Diabetic Ulcers Treated with Bi-Layered Collagen Membrane," Korean Journal of Dermatology, Vol. 47, No. 7, 2009, pp. 831-834.

[17] J. U. Shin, M. R. Roh, D. K. Rah, N. K. Ae, H, suh and K. Y. Chung, "The Effect of Succinylated Atelocollagen and Ablative Fractional Resurfacing Laser on Striae Distensae," Journal of Dermatological Treatment, Vol. 22, No. 2, 2011, pp. 113-121. doi:10.3109/09546630903476902

[18] T. J. Brown, D. Alcorn and J. R. Fraser, "Absorption of hyaluronan applied to the surface of intact skin," Journal of Investigative Dermatology, Vol. 113, No. 5, 1999, pp. 740-746. doi:10.1046/j.1523-1747.1999.00745.x

[19] M. A. Mariggiò, A. Cassano, A. Vinella, et al., "Enhancement of Fibroblast Proliferation, Collagen Biosynthesis and Production of Growth Factors as a Result of Combining Sodium Hyaluronate and Aminoacids," International Journal of Immunopathology and Pharmacology, Vol. 22, No. 2, 2009, pp. 485-492.

[20] T. Scholzen and J. Gerdes, "The Ki-67 Protein: From the Known and the Unknown," Journal of Cellular Physiology, Vol. 182, No. 3, 2000, pp. 311-322. doi:10.1002/(SICI) 1097-4652(200003)182:3<311::AID-J CP1>3.0.CO;2-9 